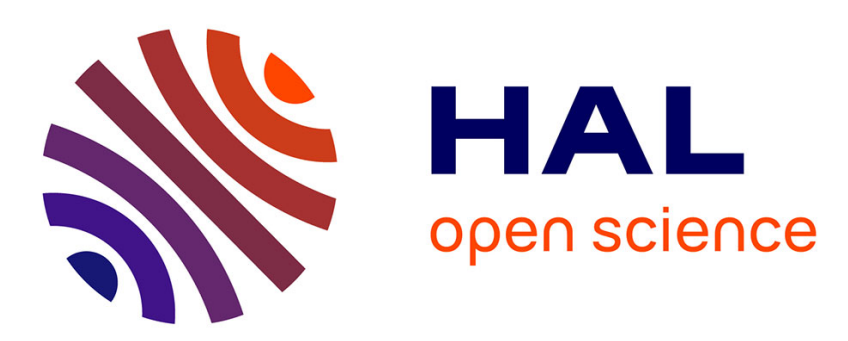

\title{
Numerical Simulations For Smouldering in a Horizontal Channel: Comparisons between variable density based formulation and incompressible one
}

\author{
Chen Yang, Gérald Debenest
}

\section{- To cite this version:}

Chen Yang, Gérald Debenest. Numerical Simulations For Smouldering in a Horizontal Channel: Comparisons between variable density based formulation and incompressible one. Combustion Science and Technology, 2014, vol. 186 ( $\mathrm{n}^{\circ}$ 12), pp. 1954-1974. 10.1080/00102202.2014.930028 . hal-01177816

\author{
HAL Id: hal-01177816 \\ https://hal.science/hal-01177816
}

Submitted on 17 Jul 2015

HAL is a multi-disciplinary open access archive for the deposit and dissemination of scientific research documents, whether they are published or not. The documents may come from teaching and research institutions in France or abroad, or from public or private research centers.
L'archive ouverte pluridisciplinaire HAL, est destinée au dépôt et à la diffusion de documents scientifiques de niveau recherche, publiés ou non, émanant des établissements d'enseignement et de recherche français ou étrangers, des laboratoires publics ou privés. 


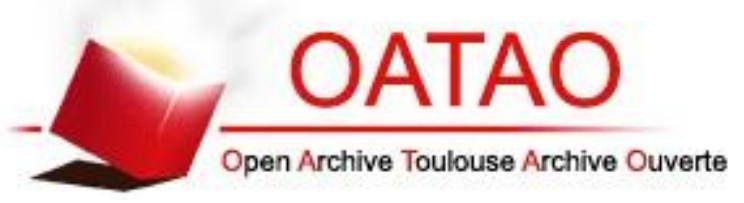

Open Archive Toulouse Archive Ouverte (OATAO)

OATAO is an open access repository that collects the work of Toulouse researchers and makes it freely available over the web where possible.

This is an author-deposited version published in: http://oatao.univ-toulouse.fr/ Eprints ID: 11839

Identification number: DOI: 10.1080/00102202.2014.930028

Official URL: http://dx.doi.org/10.1080/00102202.2014.930028

\section{To cite this version:}

Yang, Chen and Debenest, Gérald Numerical Simulations For Smouldering in a Horizontal Channel: Comparisons between variable density based formulation and incompressible one. (2014) Combustion Science and Technology, vol. 186 $\left(\mathrm{n}^{\circ} 12\right)$. pp. 1954-1974. ISSN 0010-2202

Any correspondence concerning this service should be sent to the repository administrator: staff-oatao@inp-toulouse.fr 


\title{
NUMERICAL SIMULATIONS FOR SMOLDERING IN A HORIZONTAL CHANNEL: COMPARISONS BETWEEN VARIABLE DENSITY-BASED FORMULATION AND INCOMPRESSIBLE ONE
}

\author{
C. Yang and G. Debenest \\ Université de Toulouse, INPT, UPS, IMFT (Institut de Mécanique des Fluides de \\ Toulouse), Allée Camille Soula, Toulouse, France
}

\begin{abstract}
In the present study, numerical simulations for smoldering in a horizontal channel are performed for both compressible and incompressible flows. The reactant gas is passing through the char surface, where the chemical reaction is going to take place. For the sake of simplicity, the smoldering is treated to be a single step chemical reaction. In the incompressible flow, a set of governing parameters are discussed to elucidate their influences on the process of smoldering. Furthermore, the variations of density and dynamic viscosity of gaseous mixture are taken into account in the compressible flow. The comparison between the compressible and incompressible flows reveals that the effects of local compressibility and gaseous mixture on the propagation of smoldering wave are striking.
\end{abstract}

Keywords: Compressibility; Gaseous mixture; Numerical simulations; Smoldering

\section{INTRODUCTION}

Smoldering is the flameless combustion of a liquid or solid fuel that derives heat from surface oxidation reactions. In a porous material, the local pore structure allows a gaseous reactant to filter through the solid to the reaction site. This pore scale geometry affects the flow structure and, consequently, the local mass fluxes of reactant conveyed to the sites of reaction. When the local heat content is large enough to ensure a combustion reaction, a smoldering wave can propogate through the porous medium. Due to its vast potential applications, such as porous media burner, waste incineration, catalyst regeneration, oil recovery enhancement, and many others applications (Ohlemiller, 1985; Oliveira and Kaviany, 2001; Sarathi, 1999), smoldering in porous media has attracted substantial interest.

In the open literature, there are a tremendous number of publications relevant to the smoldering in porous media. Aldushin et al. (1976) did pioneering work by conducting a one-dimensional (1D) theoretical analysis of combustion propagation wave in a porous media. Based on the asymptotic method, the structure of solution was determined for the first time. For two different structures, Schult et al. (1996) discussed solutions for reaction 
leading and reaction trailing wave structures by virtue of the asymptotic method. A socalled "reaction leading structure" occurs when the velocity of combustion front overcomes that of the heat transfer layer. In the case of a reaction trailing structure, the velocity of combustion front is slower than that of the heat transfer layer. Several experimental studies were conducted in order to prove the existence of these two reaction structures. We can report the one of Torero and Fernandez Pello (1996), where a forced forward smolder wave propagates in the reaction leading structure. A series of experiments were also done recently in the reaction trailing structure by Sennoune et al. (2012). They were able to propagate a smoldering front in reaction-trailing mode throughout an oil shale semicoke porous medium in this regime.

Regarding the numerical simulations in the microscopic geometry of porous media, two simple structure arrays of either horizontal channels or staggered cylinders are usually utilized for the computational analysis. Lu and Yortsos (2005) have studied at the local scale, the combustion in a simple dual capillary network. Gas flows through the pore network in the sites of which chemical reaction takes place. The concentrations and the temperature are kept uniform in the network sites. Furthermore, all of the transfers and couplings are modeled by effective coefficients. Hence, this cannot be regarded as a truly microscopic approach but like a mixed macroscopic and microscopic approach. We can also mention some works in filtration combustion in porous burners but only in 2D configurations once again. Uniform reactions are used inside the gas phase but no one concerns the possible heterogeneous reaction on the surface between solid and gas phases. Pioneering works can be found in Hackert et al. (1999) or Brenner et al. (2000).

Debenest et al. (2005a) proposed a 3D microscale numerical model for the simulation of smoldering in fixed beds of solid fuels. In light of a dimensional analysis, Debenest et al. (2005b) identified a set of governing parameters. Furthermore, direct and detailed numerical simulations on the microscale were performed to elucidate their influences.

However, to the best of our knowledge, microscale calculations associated with the smoldering in a porous media have yet to be done in the case of compressible flow. Most of the studies related to multicomponent and variations of density are done at the macroscopic scale.

In this case, the medium is an effective one; the entire mathematical model is built by using some effective parameters (permeability, conduction, or dispersion). Several studies were conducted in the last two decades. For instance, Moallemi et al. (1993) and Aldushin et al. (1999) have made use of the macroscale model to study the combustion of solid materials. More recently, Rein et al. (2007) presented an interesting approach to determine the kinetic scheme, which not only can be used in a smoldering process but also on combustion dynamics in porous media with and without gravity effects.

However, the effective coefficients depend on both the medium microstructure, and the flow and reaction regimes (Davit et al., 2010). Hence, their determination generally requires the solution of the full set of microscale equations in a representative sample. In addition, the validity of homogenized equations is also questionable, in view of the strong gradients on the microscopic scale (Debenest et al., 2008). As evidenced, it is necessary to investigate the effects of dilatation and gas composition on both density and viscosity to determine if an upscaled description is possible. All of the cases treated will concern both the incompressible and compositional formulas.

In this study, smoldering in the array of horizontal channels shall be revisited to investigate the effects of a set of governing equations for the incompressible flow. Then, based on the same geometry, we shall consider the variations of density and dynamic viscosity 
of gaseous mixture to discuss the independent local effects of both compressibility and gaseous mixture in the compressible flow.

\section{MATHEMATICAL MODEL AND GOVERNING EQUATIONS}

As illustrated in Figure 1a, the gas is passing through the array of horizontal channels. The gray area is the char for combustion, and the other section is the passage for gas. As a matter of fact, the realistic smoldering process in the oil shale is complex and consists of drying, devolatilization, fixed carbon oxidation, and carbonate decarbonation as pointed out by Martins et al. (2010a). Based on the relevant experimental observations conducted by Martins et al. (2010b), these reaction zones coexist and overlap with other ones. Therefore, the one-step assumption used in the present study would reduce the multiple reaction fronts to one single region. For the sake of simplicity, however, the smoldering that occurs at the interface between these two phases is treated as one single-step chemical reaction. Moreover, we make use of a 'black-box' approach to represent both the chemical reactions and the intragranular transfers of the various species involved. In this model, transposed from the 'one-film model' (Turns, 1996), the whole reaction scheme is given by a global exothermal heterogeneous reaction taking place on the surface of the reactive solid phase. This is a strong assumption in terms of chemical model, because several other studies related to the determination of kinetic scheme for oil shales, give usually a 3- or 4-step scheme. In Martins et al. (2010a), a global one-step reaction is obtained for the reaction regarding the global reaction scheme. This could be the result of a multiple reaction scheme, integrated with other front regions. A discussion with respect to all of the possible reaction schemes and scales was done in Elayeb et al. (2007). Furthermore, the combustion of a carbon residue is often treated by using a 4-effective reactions scheme, such as the one determined in Zajdlik et al. (2001).

As we would first like to investigate the differences between an incompressible and a full multicomponent approach in terms of local behaviors (maximum temperatures, front length), we choose to simplify the reactive part and focus on the transport equations model coupled with the heterogeneous reaction.

Due to the periodicity of the geometry, a unit cell would be adequate to capture the characteristics of smoldering. Moreover, for the sake of simplicity, half of a unit cell as shown in Figure $1 \mathrm{~b}$ is chosen for the calculations in this study.

Based on the mathematical model given by Debenest et al. (2005a), the governing equations used in this study for incompressible flow are indicated as follows. Note that the radiative exchange between the grains that face each other is not taken into account, although they could be significant near the reaction region. Moreover, we will consider the

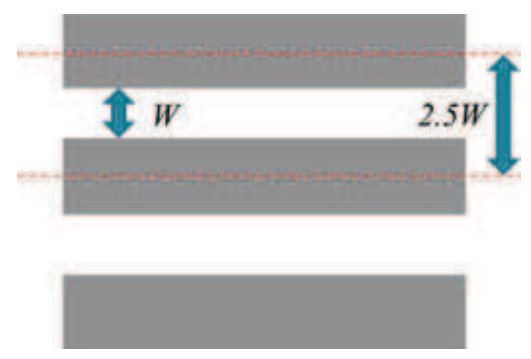

Figure 1 (a) Horizontal channels. 


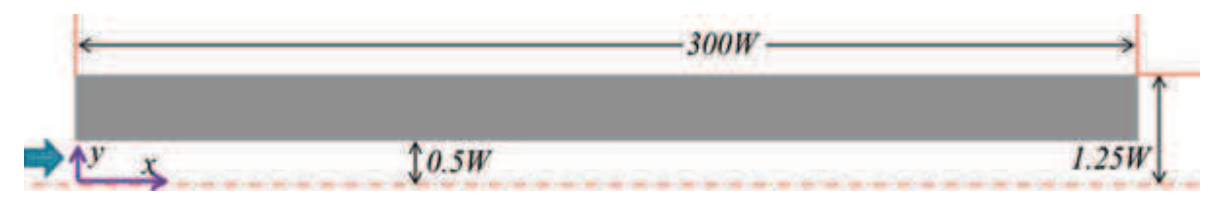

Figure 1 (b) Calculation domain.

case of low-Reynolds models to describe the flow inside the channel. This could lead to Stokes flow, but the inertial part of Navier-Stokes equations will be taken into account:

$$
\begin{gathered}
\nabla \cdot u=0 \\
\frac{\partial u}{\partial t}+\nabla \cdot(u u)=-\frac{1}{\rho} \nabla p+\frac{\mu}{\rho} \nabla^{2} u \\
\frac{\partial c_{O_{2}}}{\partial t}+\nabla \cdot J_{O_{2}}=0 \\
J_{O_{2}}=u c_{O_{2}}-D_{O_{2}} \nabla c_{O_{2}} \\
\frac{\partial\left(C_{g} T_{g}\right)}{\partial t}+\nabla \cdot J_{T g}=0 \\
J_{T g}=C_{g} u T_{g}-\lambda_{g} \nabla T_{g} \\
\frac{\partial\left(C_{s} T_{s}\right)}{\partial t}+\nabla \cdot J_{T s}=0 \\
J_{T_{s}}=-\lambda_{s} \nabla T_{s}
\end{gathered}
$$

where $c_{O_{2}}$ is the concentration of oxygen, $T_{g}$ and $T_{s}$ are the temperature of gas and solid phases, respectively. Moreover, $C_{g}$ and $C_{s}$ are the volumetric heat capacities for gas and solid phases.

Correspondingly, the boundary conditions for incompressible flow are set as follows: For $t=0$ and inlet:

$$
u=u_{i n}, \quad T_{g}=T_{s}=500 \mathrm{~K}, \text { and } c_{O_{2}}=8.376 \mathrm{~mol} / \mathrm{m}^{3}
$$

For $t=0$ and outlet:

$$
\frac{\partial u}{\partial x}=\frac{\partial T_{g}}{\partial x}=\frac{\partial T_{s}}{\partial x}=\frac{\partial c_{O_{2}}}{\partial x}=0
$$

whereas the interfacial conditions between two phases are given by:

$$
\begin{gathered}
u=0 m / s, T_{g}=T_{s}, \mathbf{n} \cdot\left(J_{T_{g}}-J_{T s}\right)=\Delta h_{c} \mathbf{n} \cdot J_{O_{2}} \\
\mathbf{n} \cdot J_{O_{2}}=S_{O_{2}} \text { and } \frac{d c_{c}}{d t}=-\frac{1}{v_{g}} \int_{\partial v_{g}} \mathbf{n} \cdot J_{O_{2}} d s
\end{gathered}
$$


where $c_{c}$ is the concentration of char, $\Delta h_{c}$ is the carbon-oxygen heat of combustion, $v_{g}$ is the grain volume, $\partial v_{g}$ is its surface, and $d s$ is a surface element. In addition, the reaction rate $S_{\mathrm{O}_{2}}$ given by Arrhenius law is indicated as follows:

$$
S_{O_{2}}=A e^{-E_{a} / R_{u} T} c_{O_{2}}
$$

where $A=10$ is the pre-exponential factor, $E_{a}=8314 \mathrm{~J} / \mathrm{mol}$ is the activation energy, and $R_{u}$ is the universal gas constant. Note that the activation energy is presumed to be constant in the wide range of temperature considered in this study. For the combustion of carbon and oxygen, however, the activation energy decreases drastically with the rise of temperature as reported by Tesner (1961). It can be found that the activation energy used for calculations has the same order of magnitude with the experimental data when the temperature is beyond $1300^{\circ} \mathrm{C}$. The choice of low activation energy is to guarantee that the reaction rate we used depends on the oxygen concentration rather than both temperature and oxygen concentration. Moreover, as we expect a quasi-steady regime when smoldering wave is developed, namely, the combustion front temperature reaches to the plateau temperature (Aldushin et al., 1999), the reactivity is affected in this case only by the presence of oxygen in a temperature range where heterogeneous combustion occurs. Then as we maintain high Damköhler values, our situation will remain similar to the realistic case with higher activation energies and pre-exponential factors.

Furthermore, the properties used in the incompressible flow are listed in Table 1.

In the case of incompressible flow, all properties of both gas and solid phases are taken to be uniform and constant. However, in the compressible flow, the variations of density and dynamic viscosity of gas phase are considered in the present study. In addition, the incoming gas is the mixture of oxygen and nitrogen in compressible flow rather than a tracer approximation for oxygen as in the incompressible flow. Owing to the utilization of a single-step chemical reaction, the generation of carbon dioxide is also considered in the compressible flow.

Based on the difference between the compressible and incompressible flows as described above, the governing equations associated with the compressible flow modified from Eqs. (1)-(5) are presented below:

$$
\begin{gathered}
\frac{\partial \rho}{\partial t}+\nabla \cdot J_{\rho}=0 \\
J_{\rho}=\rho u \\
\frac{\partial \rho u}{\partial t}+\nabla \cdot(\rho u u)=-\nabla\left(p+\frac{2}{3} \mu \nabla \cdot u\right)+\nabla \cdot\left[\mu\left(\nabla u+(\nabla u)^{T}\right)\right]
\end{gathered}
$$

\begin{tabular}{|c|c|c|c|c|c|}
\hline \multicolumn{3}{|l|}{ Solid } & \multicolumn{3}{|c|}{ Gas } \\
\hline$\rho_{\mathrm{s}}$ & 2100 & $\mathrm{~kg} / \mathrm{m}^{3}$ & $\rho_{\mathrm{g}}$ & 0.3 & $\mathrm{~kg} / \mathrm{m}^{3}$ \\
\hline$c_{p s}$ & 800 & $\mathrm{~J} / \mathrm{kgK}$ & $c_{p g}$ & 1200 & $\mathrm{~J} / \mathrm{kgK}$ \\
\hline$\lambda_{\mathrm{s}}$ & 1 & $\mathrm{~W} / \mathrm{mK}$ & $\lambda_{\mathrm{g}}$ & 0.025 & $\mathrm{~W} / \mathrm{mK}$ \\
\hline$\Delta \mathrm{h}_{\mathrm{c}}$ & 391.9 & $\mathrm{~kJ} / \mathrm{mol}$ & $\mathrm{D}_{\mathrm{O}_{2}}$ & $2 \times 10^{-4}$ & $\mathrm{~m}^{2} / \mathrm{s}$ \\
\hline
\end{tabular}

Table 1 Properties in the incompressible flow 


$$
\begin{gathered}
\frac{\partial \rho Y_{O_{2}} / M_{W_{O_{2}}}}{\partial t}+\nabla \cdot J_{O_{2}}=0 \\
J_{O_{2}}=\left(\rho u Y_{O_{2}}-\rho D_{O_{2}} \nabla Y_{O_{2}}\right) / M_{W_{O_{2}}} \\
\frac{\partial \rho Y_{C O_{2}} / M_{W_{C O_{2}}}+\nabla \cdot J_{C O_{2}}=0}{J_{C O_{2}}=\left(\rho u Y_{C O_{2}}-\rho D_{C O_{2}} \nabla Y_{C O_{2}}\right) / M_{W_{C O_{2}}}} \\
\frac{Y_{N_{2}}=1-Y_{O_{2}}-Y_{C O_{2}}}{\partial t}+\nabla \cdot J_{T g}=0 \\
J_{T g}=C_{g} u T_{g}-\lambda_{g} \nabla T_{g} \\
\frac{\partial\left(C_{s} T_{s}\right)}{\partial t}+\nabla \cdot J_{T s}=0 \\
J_{T_{s}}=-\lambda_{s} \nabla T_{s}
\end{gathered}
$$

where the $Y_{i}$ is the mass fraction of pure gas, and the variations of density and dynamic viscosity of gas phase are achieved by the following two equations:

$$
\rho=\frac{p}{R_{u} T_{g} \sum_{i=1}^{N}\left(Y_{i} / M_{W_{i}}\right)}
$$

and

$$
\mu=\sum_{i=1}^{N} X_{i} \mu_{i}
$$

where $M_{W_{i}}$ and $X_{i}$ are the molecular weight and molar fraction of pure gas, respectively. In addition, the dynamic viscosity of pure gas $\mu_{i}$ can be calculated as follows:

$$
\mu_{i}=\frac{A T_{g}^{B}}{1+C / T_{g}+D / T_{g}^{2}}
$$

The corresponding coefficients for the pure gas in Eq. (19) referred from Chapman and Cowling (1939) are listed in Table 2.

The boundary conditions for compressible flow are set as follows:

For $t=0$ and inlet:

$$
\begin{aligned}
& u=u_{i n}, T_{g}=T_{s}=500 \mathrm{~K}, Y_{O_{2}}=0.8935,0.1787,0.0596, Y_{C_{2}}=0, \\
& Y_{N_{2}}=1-Y_{O_{2}}-Y_{C O_{2}}
\end{aligned}
$$


Table 2 Coefficients in Eq. (19)

\begin{tabular}{lcccc}
\hline & $A$ & $B$ & $C$ & $D$ \\
\hline $\mathrm{O}_{2}$ & $1.10 \times 10^{-6}$ & 0.56 & 96.3 & 0 \\
$\mathrm{CO}_{2}$ & $2.14 \times 10^{-6}$ & 0.46 & 290 & 0 \\
$\mathrm{~N}_{2}$ & $6.56 \times 10^{-7}$ & 0.61 & 54.7 & 0 \\
\hline
\end{tabular}

For $t=0$ and outlet, a no-flux condition is imposed for all transport equations:

$$
\frac{\partial u}{\partial x}=\frac{\partial T_{g}}{\partial x}=\frac{\partial T_{s}}{\partial x}=\frac{\partial Y_{O_{2}}}{\partial x}=\frac{\partial Y_{N_{2}}}{\partial x}=\frac{\partial Y_{C O_{2}}}{\partial x}=0
$$

whereas the interfacial conditions between two phases are given by:

$$
\begin{gathered}
u=0 m / s, T_{g}=T_{s}, \mathbf{n} \cdot\left(J_{T_{g}}-J_{T s}\right)=\Delta h_{c} \mathbf{n} \cdot J_{O_{2}}, \mathbf{n} \cdot J_{O_{2}}=S_{O_{2}}, \\
\mathbf{n} \cdot J_{\mathrm{CO}_{2}}=S_{\mathrm{CO}_{2}}=S_{O_{2}} \times M_{W_{\mathrm{CO}_{2}}} / M_{W_{O_{2}}}, \mathbf{n} \cdot J_{\rho}=S_{\rho}=S_{\mathrm{CO}_{2}}-S_{O_{2}}, \\
\frac{d c_{c}}{d t}=-\frac{1}{v_{g}} \int_{\partial v_{g}} \mathbf{n} \cdot J_{O_{2}} d s
\end{gathered}
$$

In the present study, a set of governing parameters used for the analysis are presented as follows:

$$
P e=\frac{u_{B} W}{D_{O_{2}}}, \Delta=\frac{C_{g} \Delta c_{c}}{C_{s} \Delta c_{O_{2}}}, T_{a d}=\frac{\varepsilon_{s} \Delta c_{c} \Delta h_{c}}{\varepsilon_{g} C_{g}+\varepsilon_{s} C_{s}}, T_{p}=\frac{T_{a d}}{|\Delta-1|}
$$

where $\varepsilon_{g}$ and $\varepsilon_{s}$ are porosities of gas and solid phases, $T_{a d}$ and $T_{p}$ are the adiabatic temperature and plateau temperature, respectively. In addition, the adiabatic temperatures used for both compressible and incompressible flows are indicated in Table 3.

In the case of incompressible approach, we change $\Delta$ values by adjusting the carbon content, $\Delta c_{c}$. As the temperature level will not change any gas phase properties, the variation of $\Delta$ has no impact on the solution. However, the variations of temperature and composition will modify all physical properties of the gas phase in the case of compressible approach. Thus, changing $\Delta$ value is a major problem concerned in this study. We choose to adjust $\Delta$ by changing $\Delta c_{O_{2}}$. Consequently, no modifications of adiabatic temperature appear in the compressible approach. Hence, we define a temperature level of $911.69 \mathrm{~K}$ for all $\Delta$ values. This is only done to define a reference point, and then to have a comparison

Table 3 The adiabatic temperatures used for compressible and incompressible flows

\begin{tabular}{lccrrr}
\hline & $\Delta$ & 0.1 & 0.5 & 1.0 & \multicolumn{1}{c}{1.5} \\
\hline Incompressible & $T_{a d}(\mathrm{~K})$ & 911.69 & 4558.45 & 9116.9 & $13,675.36$ \\
Compressible & $T_{a d}(\mathrm{~K})$ & 911.69 & 911.69 & 911.69 & 911.69 \\
\hline
\end{tabular}


between all compressible cases (temperature profiles and concentrations depending on $\Delta$ values) but also to be able to compare compressible and incompressible approaches.

It can be shown by a simple 1D continuous model from Dosanjh et al. (1987) or in Debenest et al. (2005b) that if $\Delta>1$ (large fuel or low oxidizer concentrations), heat is transferred from the upstream to the downstream region. An asymptotic temperature profile with a plateau $T_{p}$ on the downstream side is expected. In the opposite case $\Delta<1$, the plateau is expected on the upstream side. The intermediate case $\Delta=1$ corresponds to the ultimate superadiabatic situation, where heat remains in the vicinity of the reaction front. Note that $\Delta$ can also be expressed as a function of the heat capacities and of the volumetric concentrations of carbon $\left(c_{c}\right.$, in the solid) and oxidizer $\left(c_{O_{2}}\right.$, in the gas).

\section{CODE VERIFICATIONS}

The foregoing sets of governing equations are solved numerically by using the OpenFOAM Version 2.1.1 package. Since the process of smoldering is transient, PIMPLE algorithm designed for the large time-step computations is chosen to be the numerical scheme. The transient terms of the foregoing sets of governing equations were discretized using the first-order Euler implicit scheme. For the convergence criteria, the residuals of all variables are less than $10^{-12}$. Moreover, we have performed a sensitivity analysis to the grid size. Then, all of the calculation results in the present study are independent of the grid size. The grid size utilized in this study is $300 \times 100$.

As depicted in Figure 2, the fully developed velocity profile of a cross-section of the channel is compared with the corresponding exact solution, which is expressed as $\frac{u}{u_{B}}=\frac{3}{2}\left(1-\left(\frac{y}{0.5 W}\right)^{2}\right)$. It can be seen that the agreement between two velocity profiles is good. According to the prediction of Debenest et al. (2005b), the transversally averaged temperature is supposed to reach the plateau temperature calculated by Eq. (23d). In Figure 3, the transversally averaged temperature comes to the plateau temperature when the location of the combustion front is $70 \mathrm{~W}$ at the estimated time, which can be easily deduced from the formulation of combustion front velocity $U_{F}=\frac{\varepsilon_{g} \Delta c_{O_{2}}}{\varepsilon_{s} \Delta c_{c}} u_{\text {in }}$ proposed by Debenest et al. (2005b). Moreover, as shown in Figure 4, the increase of maxmuim transversally averaged temperature for the case of $\Delta=1$ is linear to the square root of time, which is consistent with the conclusion addressed by Aldushin et al. (1999). In summary, the sets of governing equations are verified and are suitable for the calculations in this study.

\section{RESULTS AND DISCUSSION}

Several tests were made in order to check the accuracy of the model. These are summarized in Table 4.

For all of the calculations, $P e$ and $\Delta$ reported in Table 4 are the results obtained for averaged temperature divided by the adiabatic one. If the results are correct, it should converge to the value of $1 /|\Delta-1|$, according to Aldushin et al. (1976). We see a good agreement with the theory for $P e=1,10 ; \Delta=0.1$ with a profile of averaged temperature coming to the expected temperature. But, for $\Delta$ values equal to 0.5 and 1.5 , a difference of less than $3 \%$ is observed. This is the asymptotic value reached numerically and several things could explain that. The main direction we have today is the grid size in $y$ direction as the reaction term depends strongly on the concentration of oxygen. But, as evidenced, 


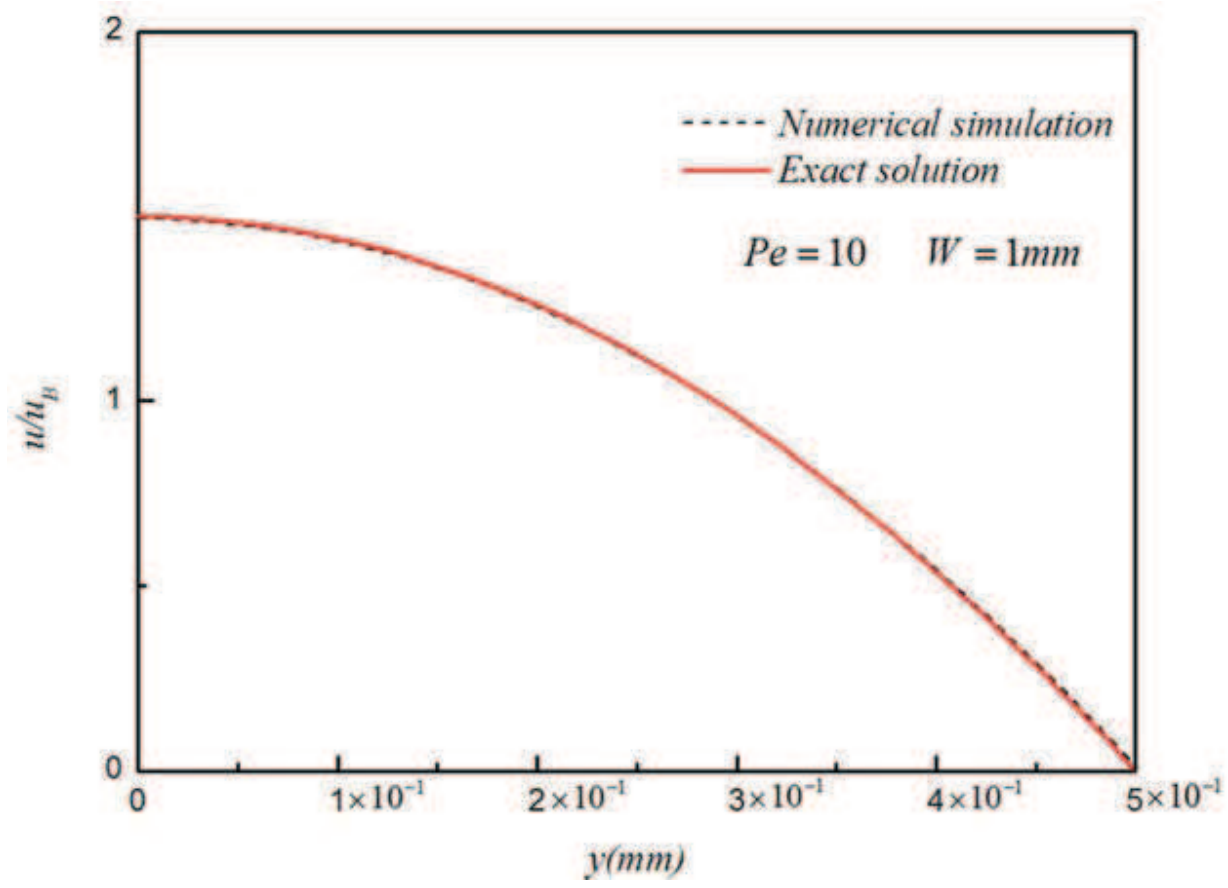

Figure 2 Comparison of fully developed velocity profile in a channel with the exact solution.

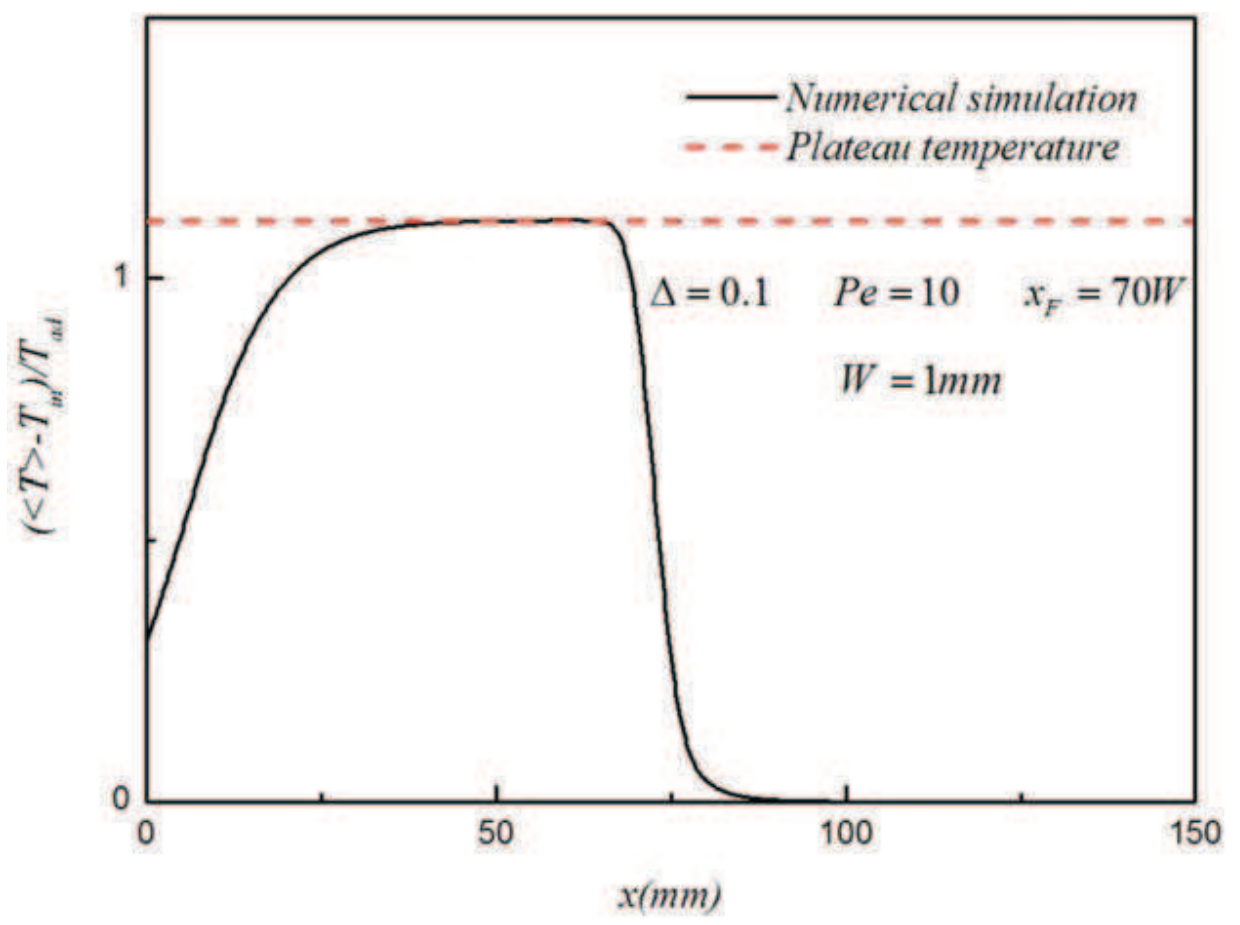

Figure 3 Profile of the transversally averaged temperature in the horizontal channel.

we can assume that the numerical tool is valid, and could be a starting point for further investigations. We are now going to see more details of the results.

As illustrated in Figures 5, 6, and 7, the profiles of transversally averaged temperatures of gas and solid phases are presented for three different $\Delta \mathrm{s}$. The Peclet number, $P e$, is kept constant and equal to 10 . Different $\Delta \mathrm{s}$ can be interpreted as different char concentration in the solid or different oxidizer concentration in the gas. In these three figures, it 


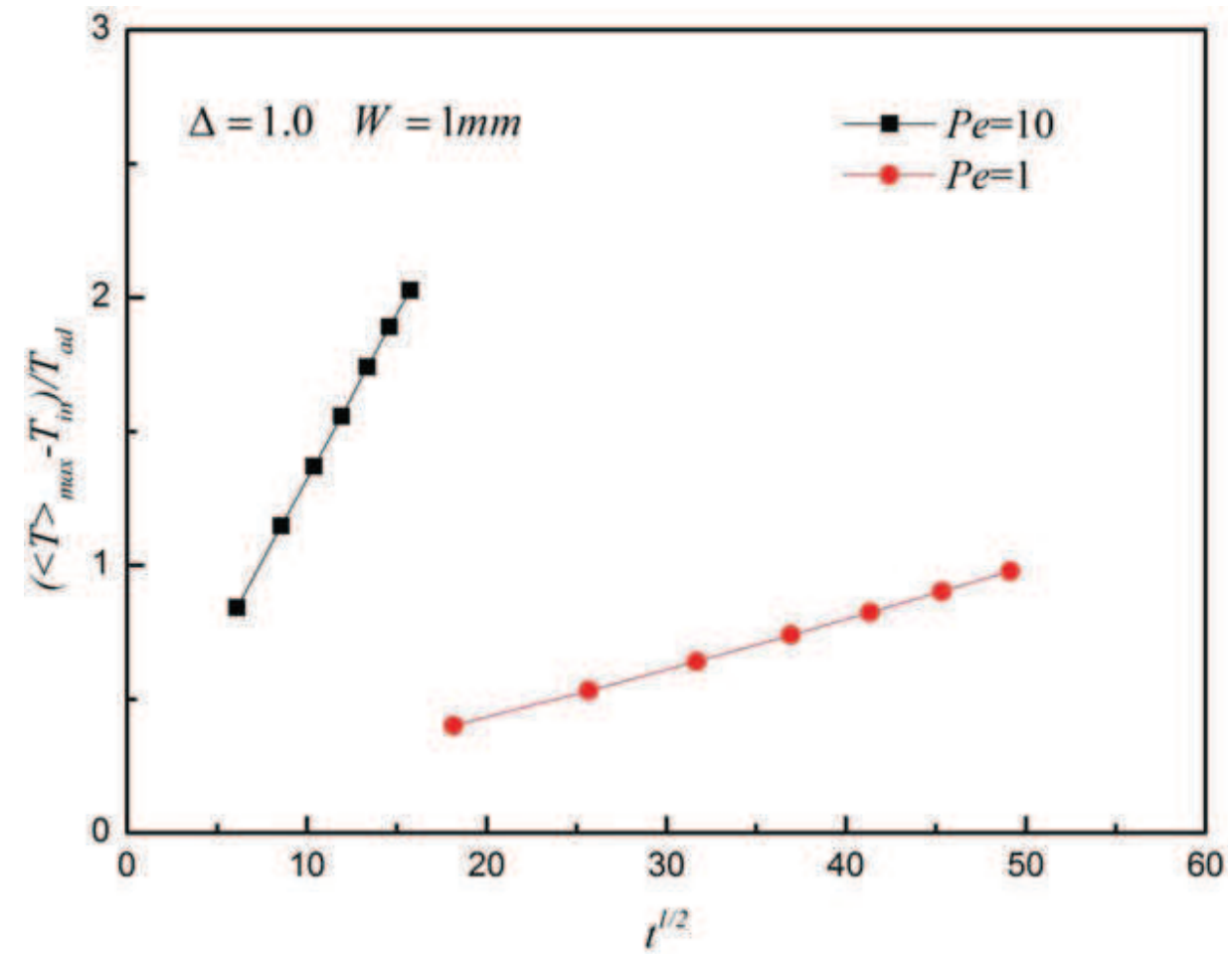

Figure 4 The relationship between the increase of maximum transversally averaged temperature and square root of time for the case of $\Delta=1$.

Table 4 Several cases in the present study

\begin{tabular}{|c|c|c|c|c|c|c|}
\hline & \multicolumn{2}{|c|}{0.1} & \multicolumn{2}{|c|}{0.5} & \multicolumn{2}{|c|}{1.5} \\
\hline & \multicolumn{6}{|c|}{$T_{p} / T_{a d}$} \\
\hline & Numerical & Analytical & Numerical & Analytical & Numerical & Analytical \\
\hline 1 & 1.11 & 1.111 & $x$ & 2.0 & $\times$ & 2.0 \\
\hline 10 & 1.11 & 1.111 & 1.976 & 2.0 & 1.97 & 2.0 \\
\hline
\end{tabular}

can clearly be seen that the smoldering wave propagates as the time goes by, and eventually reaches a steady regime. The amount of time depends on the flow regime as seen in Debenest et al. (2005b).

In Figure 5, the steady regime is obtained after $40 \mathrm{~W}$. We can see a difference in the averaged temperature profiles between the gas and solid phase. At the inlet, the gas phase needs several $\mathrm{W}$ before being at the equilibrium with the solid phase. A peak is observed in the gas phase in the reaction region. Then, the gas phase appears to be hotter than the solid phase. This is due to the convective part in Eq. (4a).

In Figure 6, the steady regime is obtained after $160 \mathrm{~W}$. The maximum averaged temperature is really close to the analytical solutions. We also observe that the heat content stays closer to the reaction region than in the previous case. This is coming from the increasing $\Delta$ value. Heat transport velocity is now close to the reaction front velocity and the two waves propagate at nearly the same speed. 


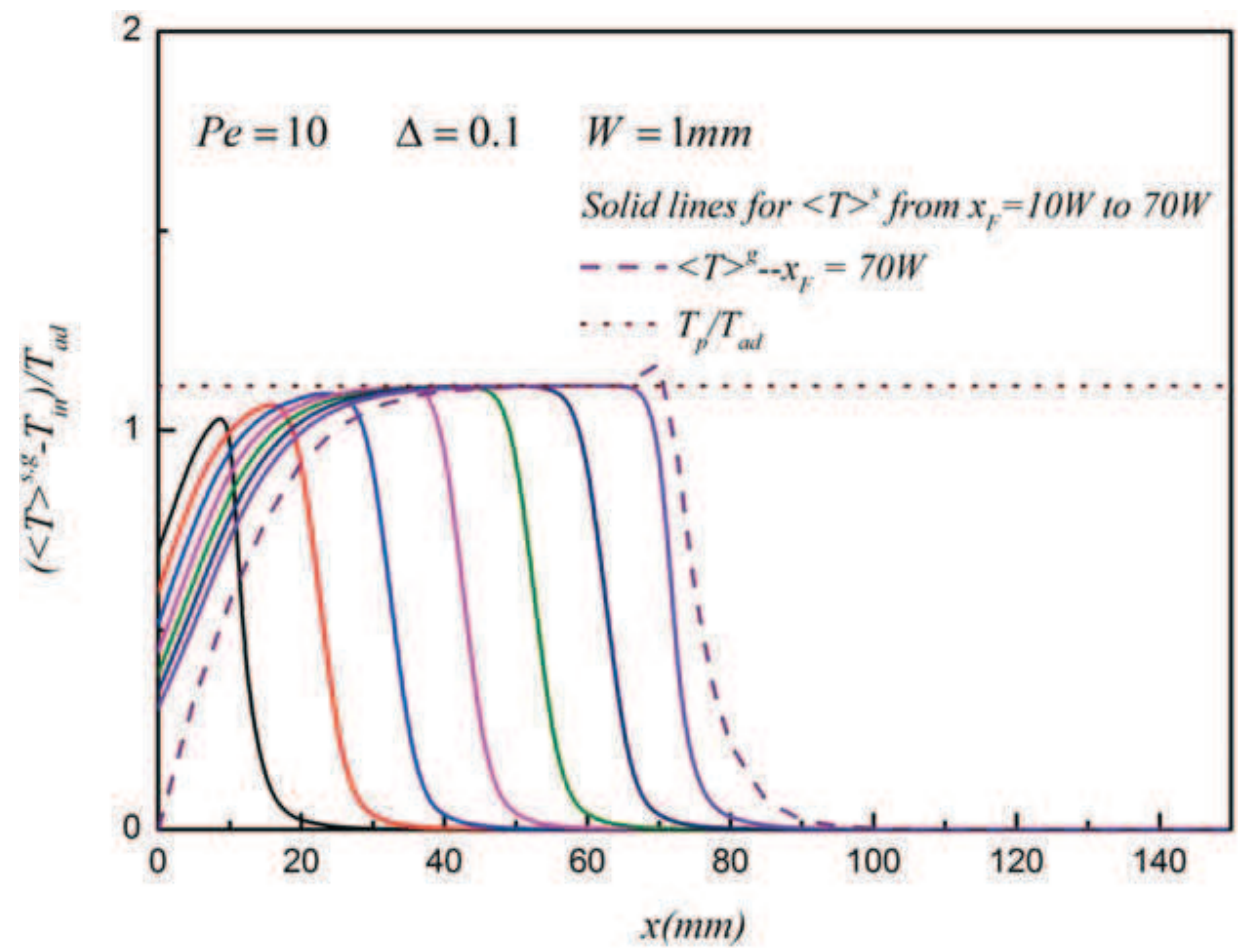

Figure 5 Profiles of the transversally averaged temperature of fluid and solid phases for $\Delta=0.1$ and $P e=10$ in the incompressible flow.

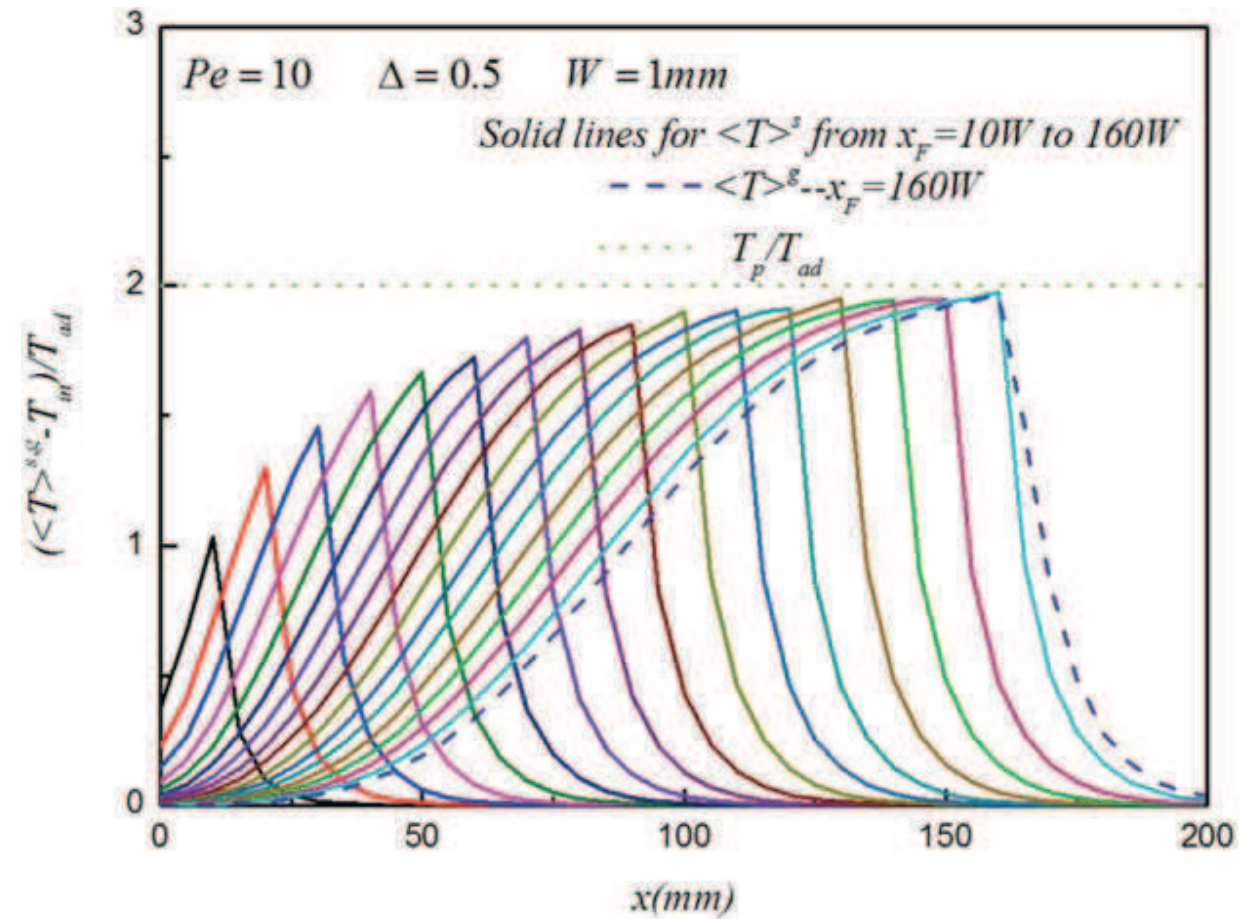

Figure 6 Profiles of the transversally averaged temperature of fluid and solid phases for $\Delta=0.5$ and $P e=10$ in the incompressible flow. 


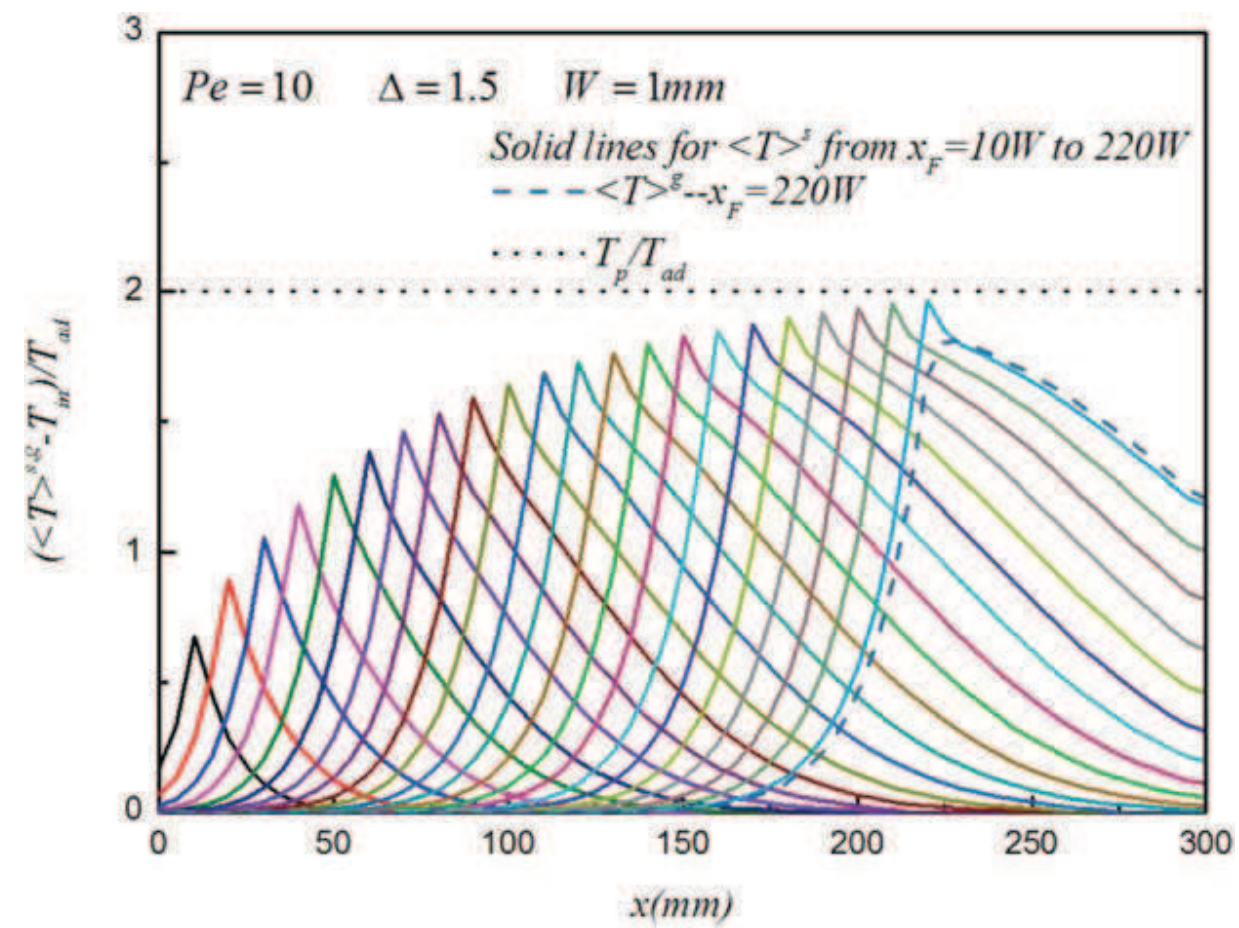

Figure 7 Profiles of the transversally averaged temperature of fluid and solid phases for $\Delta=1.5$ and $P e=10$ in the incompressible flow.

In Figure 7, the steady regime is obtained after $220 \mathrm{~W}$. The maximum temperature is close to $2 T_{a d}$. The thermal wave propagates quicker than the reaction wave, so the heat content accumulates after the reaction region.

In these two preceding cases, we see the differences in transversally averaged temperature profiles between gas and solid phases by analogy with that indicated in Figure 5. Moreover, propagation waves of these two cases reach the same plateau temperature calculated from Eq. (23d), yet the difference between smoldering structures of two cases are obvious. For the case of $\Delta=0.5$ and $P e=10$, enough heat is left behind to preheat the incoming gas since the velocity of heat transport is close to that of reaction front. As the further increase of $\Delta$, the reaction front velocity overwhelms the heat transport velocity, which explains that most of heat is taken to the downstream as shown in Figure 7 for the case $\Delta=1.5$ and $P e=10$. It also should be noted that it is the first time to obtain the stationary regimes for both the cases of $\Delta=0.5$ and $\Delta=1.5$ in the literature.

In Figure 8, the case $P e=1$ and $\Delta=0.1$ is given. After $50 \mathrm{~W}$, the steady regime is observed and the averaged temperature reaches the expected value. When the combustion front comes to $70 \mathrm{~W}$, the averaged profiles of gas temperature and solid temperature are plotted. No significant differences exist, except in the inlet zone where the gas phase enters at $500 \mathrm{~K}$. In the front region, a peak temperature exists in the gas phase. This comes from the reaction at the interface; a small amount of heat released to the gas phase leads to an increase of temperature due to small gas heat capacity. After a short distance equal to 1 or $2 \mathrm{~W}$, the averaged gas temperature rapidly approaches to the averaged one of the solid phase. The same $\Delta$ value with a $P e=10$, gives us a strong difference in space between the two temperatures. Because of the small value of $P e$ and especially of thermal $P e$ value as explained in Debenest et al. (2008), in this case there are no differences. 


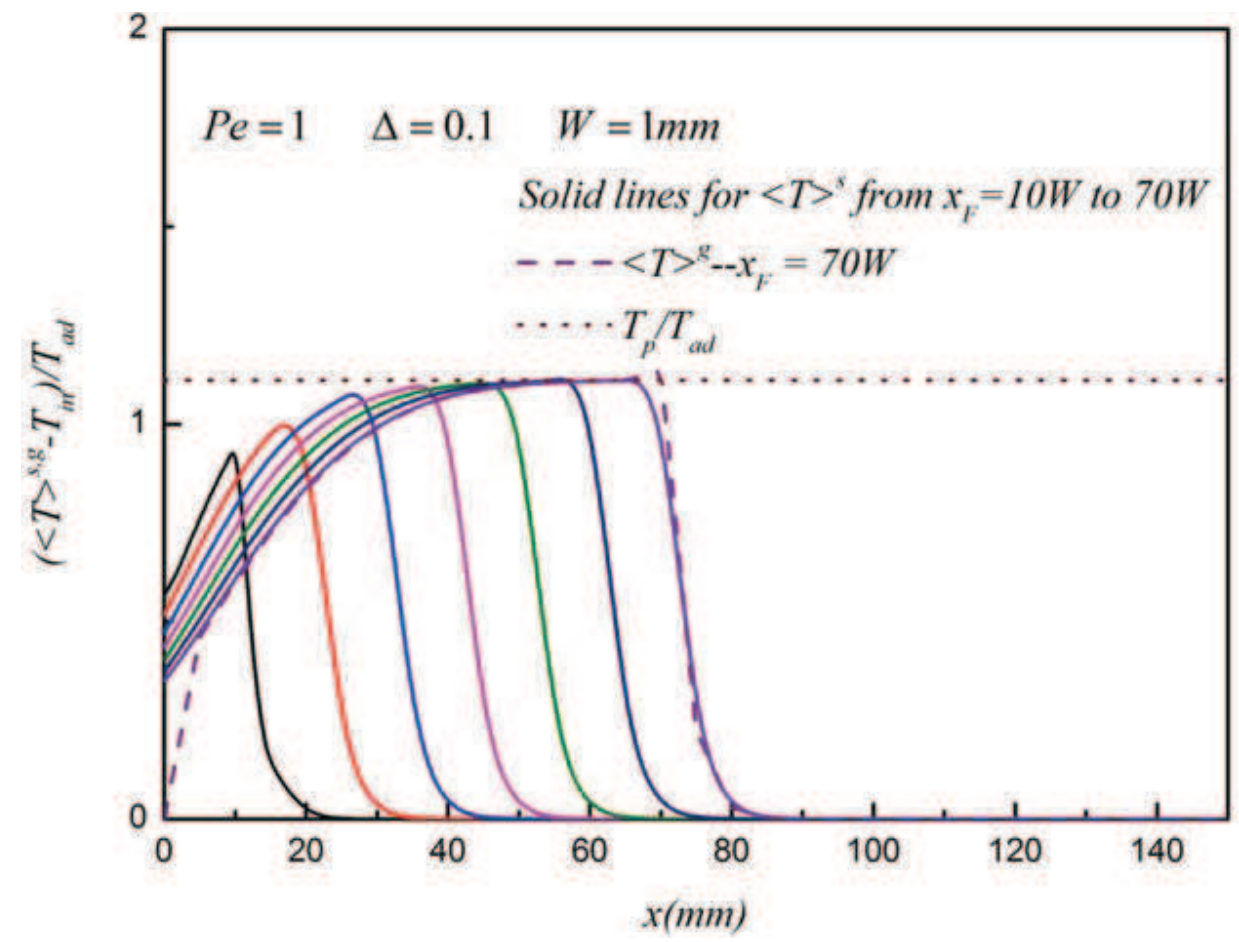

Figure 8 Profiles of the transversally averaged temperature of fluid and solid phases for $\Delta=0.1$ and $P e=1$ in the incompressible flow.

Now let's examine the compressible flows as introduced in Eqs. (10) to (16). The last part of this article will be dedicated to the comparison between incompressible and compressible flows and quantification of the effects of composition and temperature with respect to density and dynamic viscosity. Several tests will be made to be compared with our incompressible case by varying the $P e$ number and $\Delta$ values.

In Figures 9 and 10, the cases of $P e=1$ and 10 for the $\Delta=0.1$ are presented for compressible flow. By comparing these two figures with Figures 5 and 8, it can be seen that the variations of density and dynamic viscosity of the gas phase have no remarkable influence on the propagation of smoldering wave for the case of $P e=10$ and $\Delta=0.1$. Further investigations seem to be necessary, such as more accurate chemical reaction schemes, variation of thermal capacity of gas phase, etc.

As shown in Figures 11 and 12, differences of density and dynamic viscosity of the gas phase between compressible and incompressible flows are plotted in the direction of smoldering wave propagation. The comparison results clearly indicate that the difference is significant; especially in the chemical reaction region. This is because the variations of density and dynamic viscosity of the gas phase are highly temperature and compositiondependent.

In Figures13-15, the profiles of the transversally averaged temperature of solid phase for $P e=10$ in the incompressible and compressible flows are presented. Three different cases, namely, $\Delta=0.1,0.5$, and 1.5 , are discussed. As illustrated in these three figures, the differences between the incompressible and compressible flows are significant. It can be clearly seen that the cases of compressible flow require longer distance to reach the equilibrium regime. Moreover, the maximum temperature of compressible flow in the direction of smoldering propagation wave is lower than that of incompressible flow. The temperature 


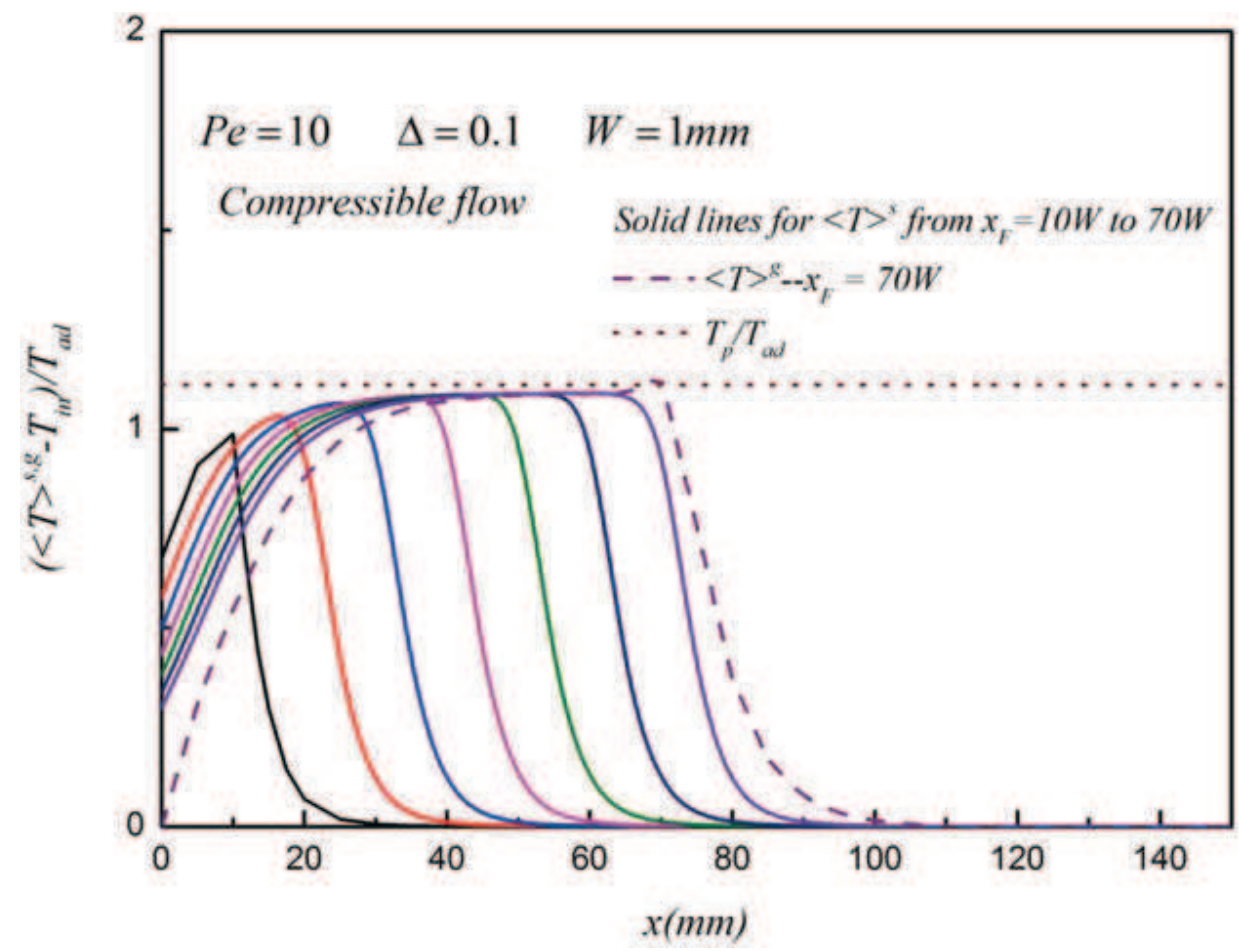

Figure 9 Profiles of the transversally averaged temperature of fluid and solid phases for $\Delta=0.1$ and $P e=10$ in the compressible flow.

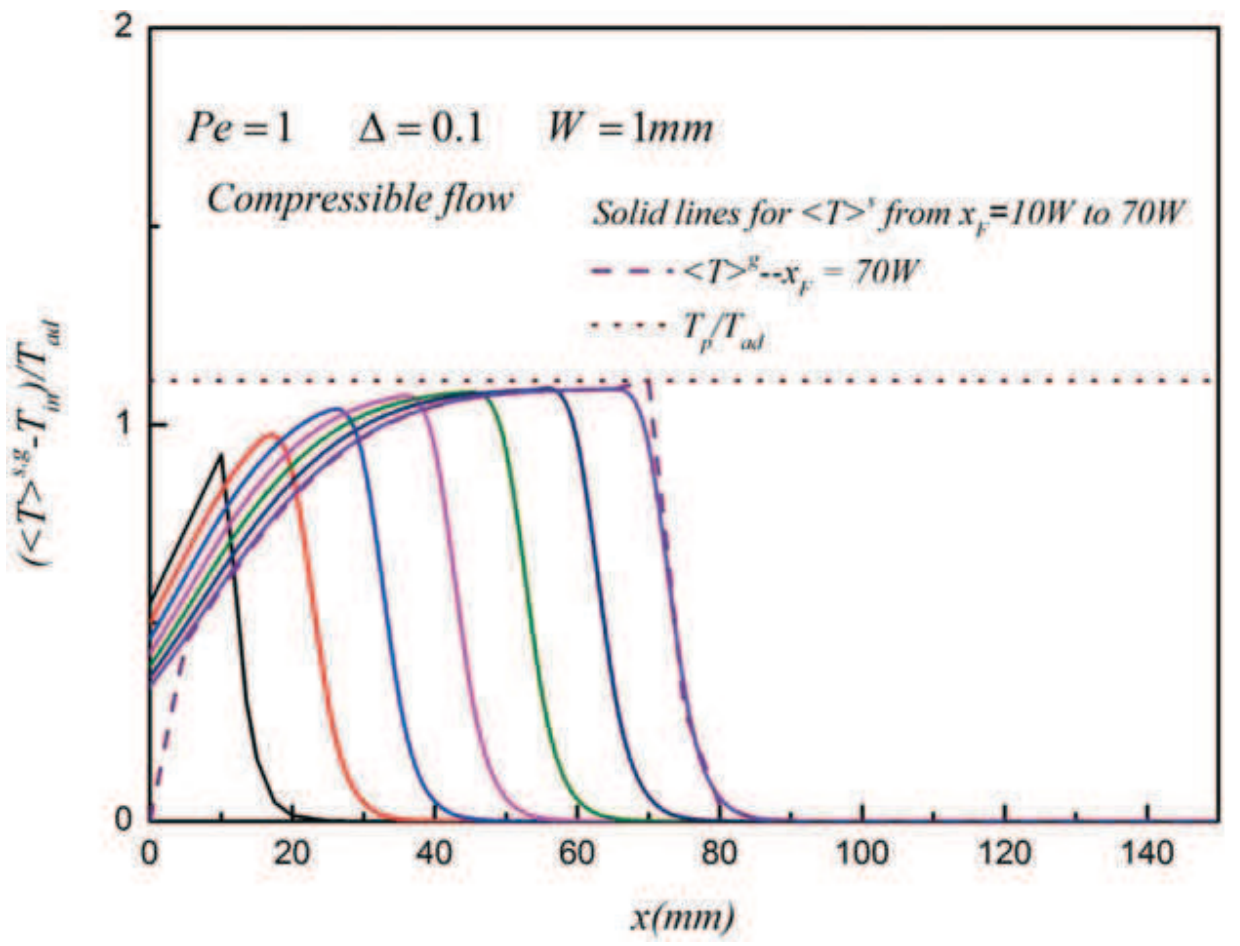

Figure 10 Profiles of the transversally averaged temperature of fluid and solid phases for $\Delta=0.1$ and $P e=1$ in the compressible flow. 


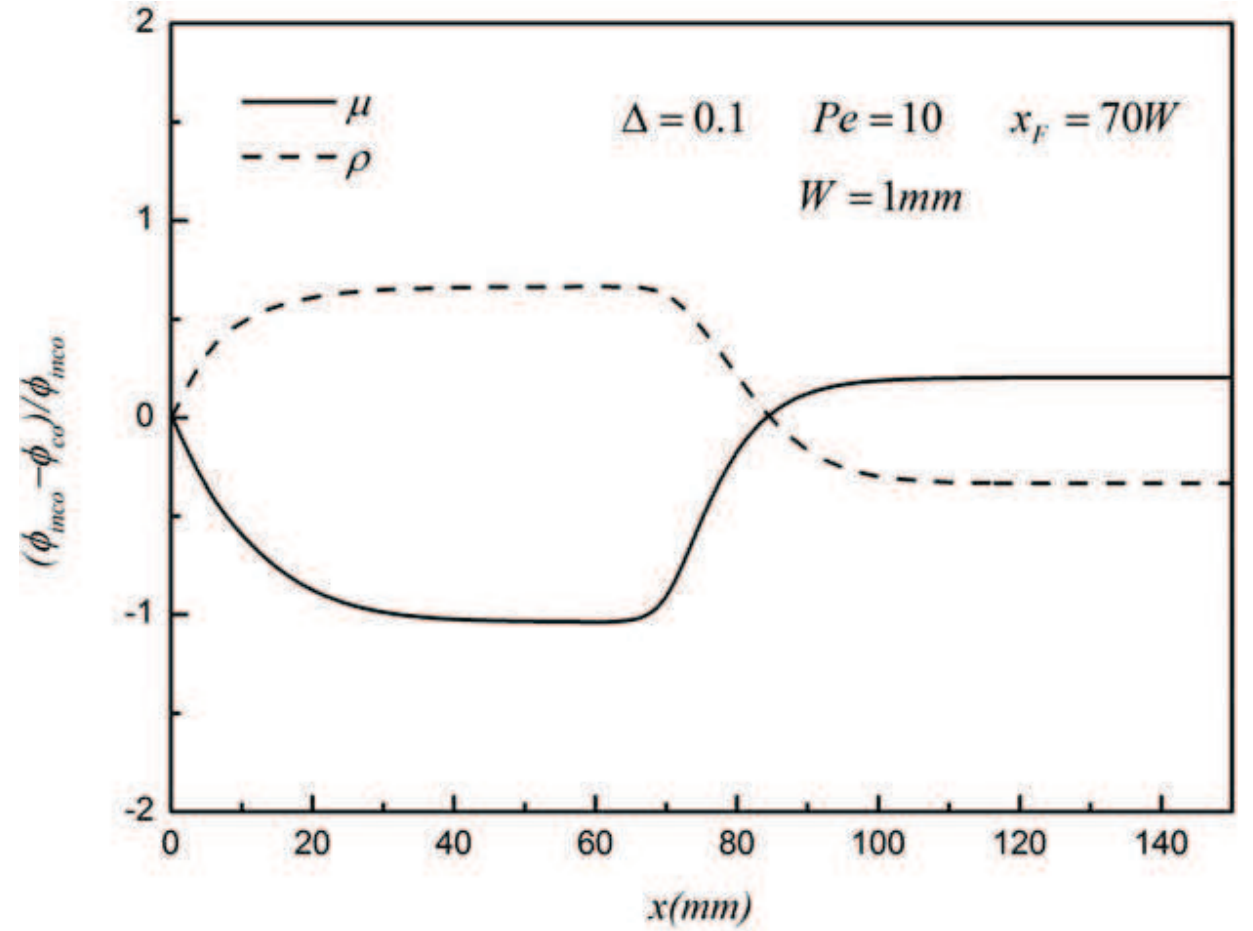

Figure 11 Comparisons between the compressible and incompressible flows for $\Delta=0.1$ and $P e=10$.

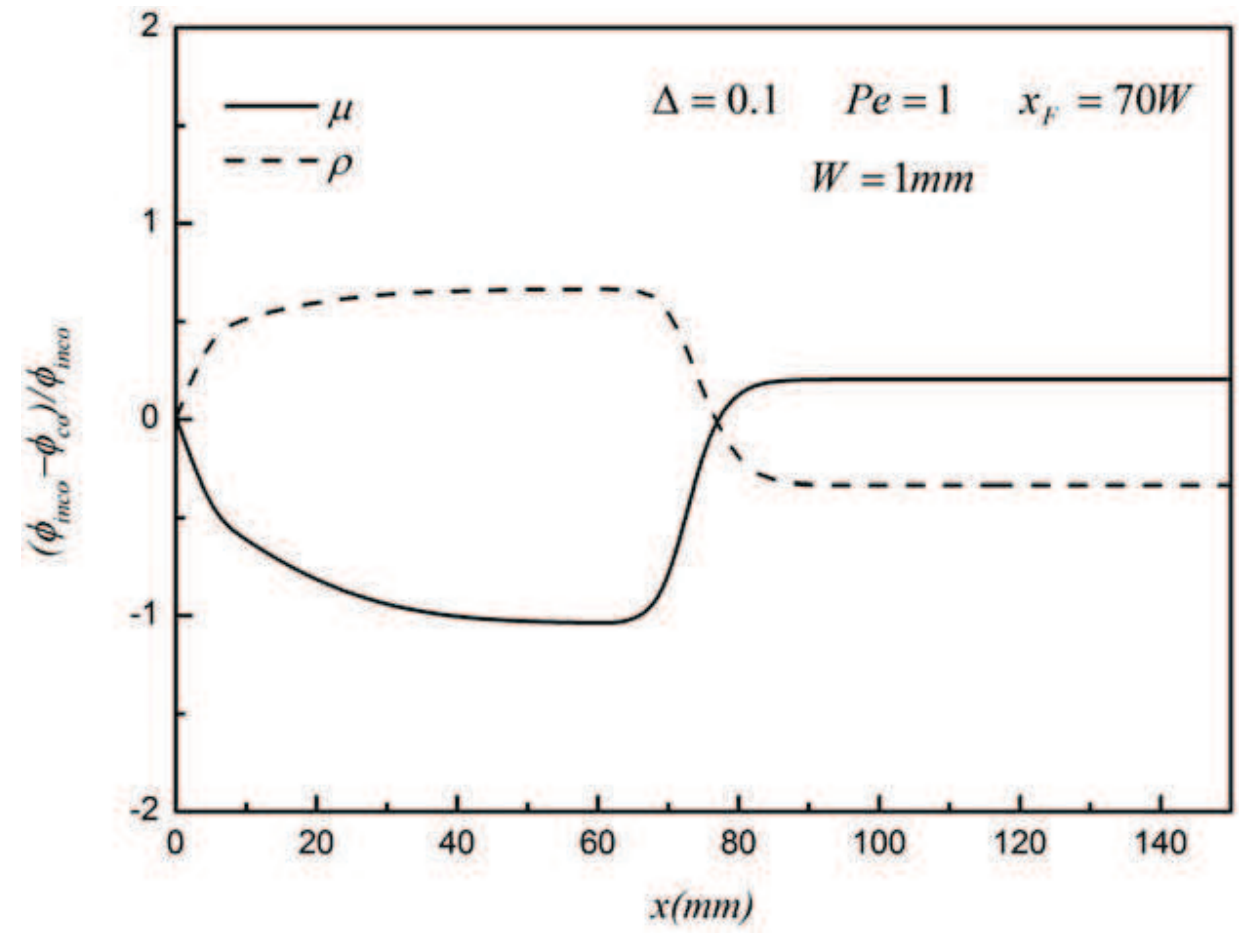

Figure 12 Comparisons between the compressible and incompressible flows for $\Delta=0.1$ and $P e=1$. 


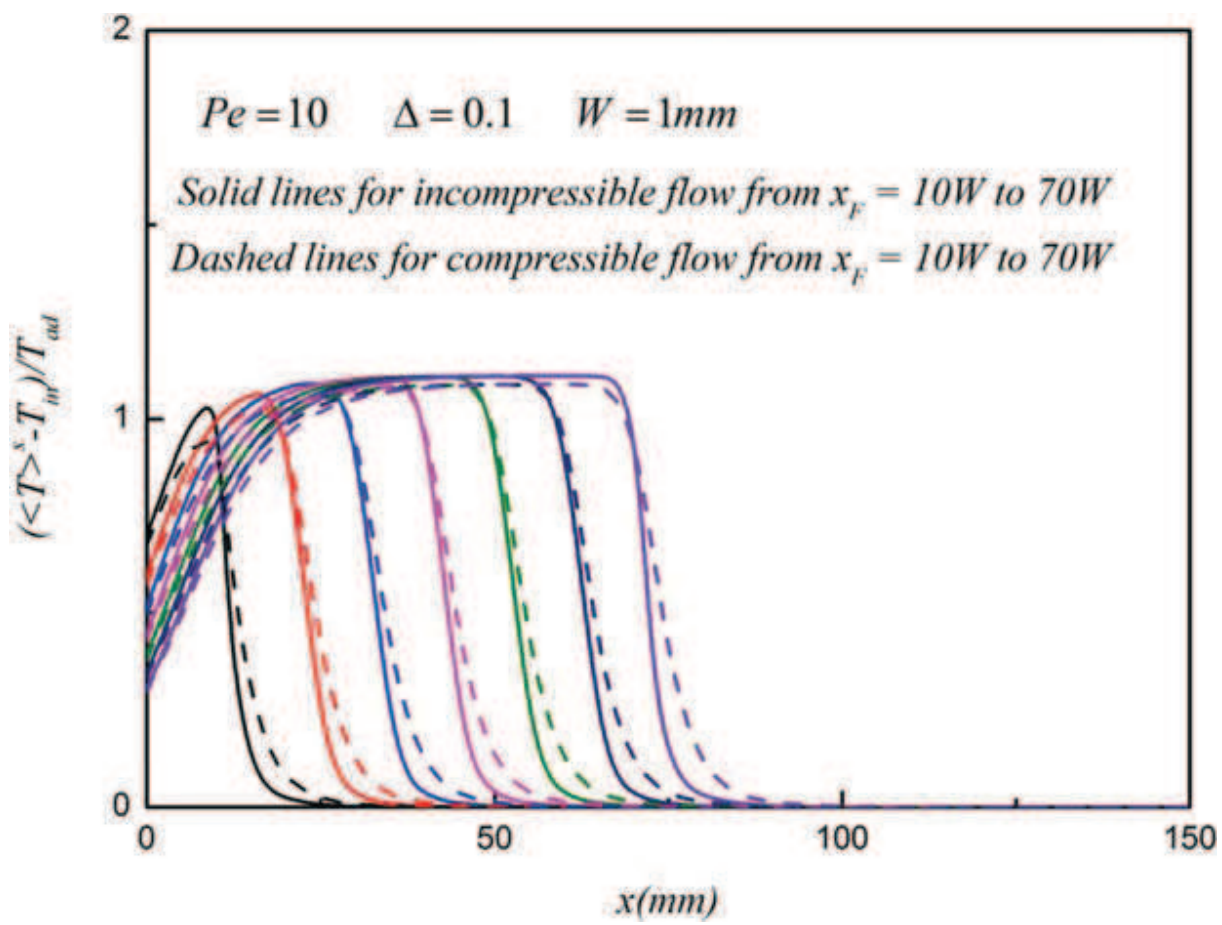

Figure 13 Profiles of the transversally averaged temperature of solid phase for $\Delta=0.1$ and $P e=10$ in the incompressible and compressible flows.

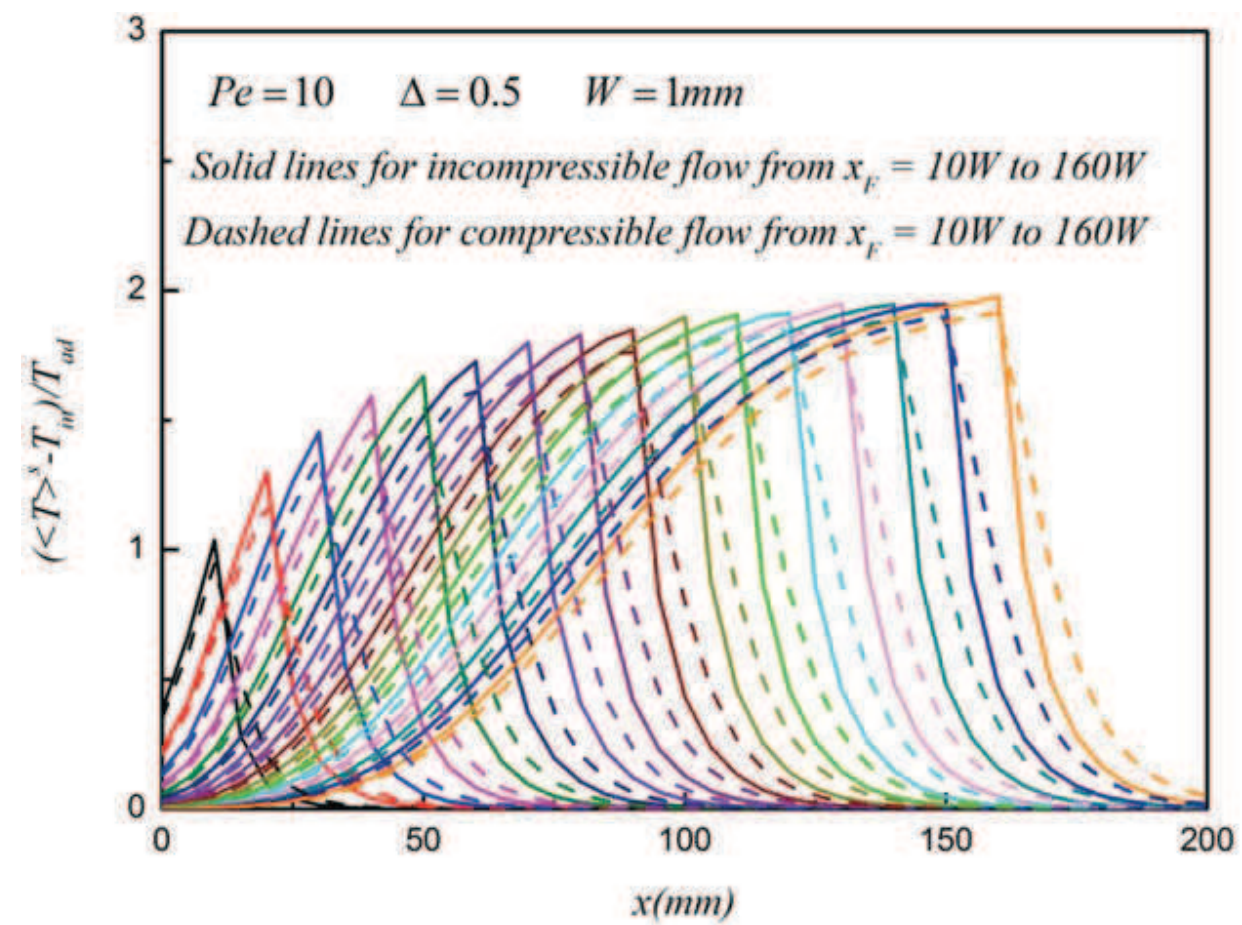

Figure 14 Profiles of the transversally averaged temperature of solid phase for $\Delta=0.5$ and $P e=10$ in the incompressible and compressible flows. 


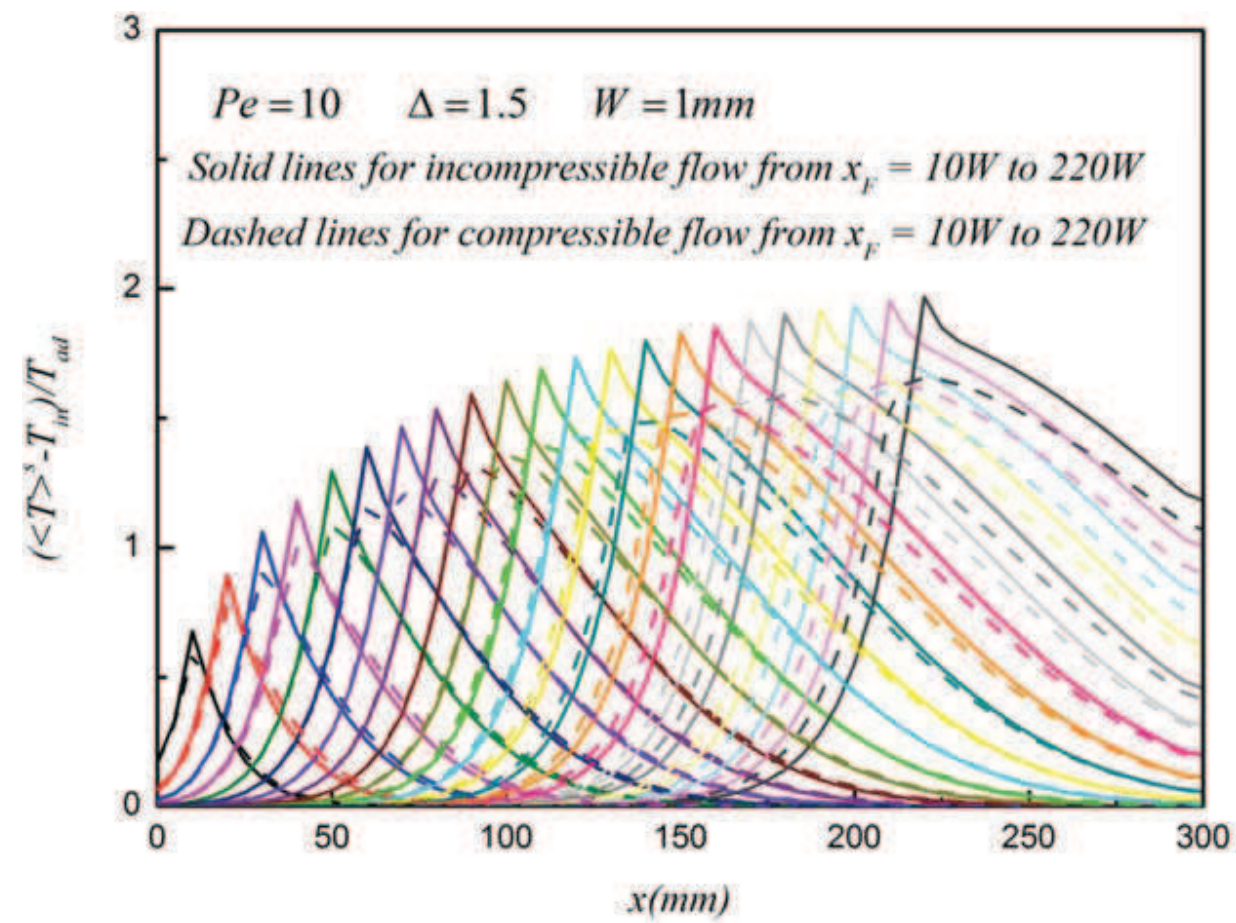

Figure 15 Profiles of the transversally averaged temperature of solid phase for $\Delta=1.5$ and $P e=10$ in the incompressible and compressible flows.

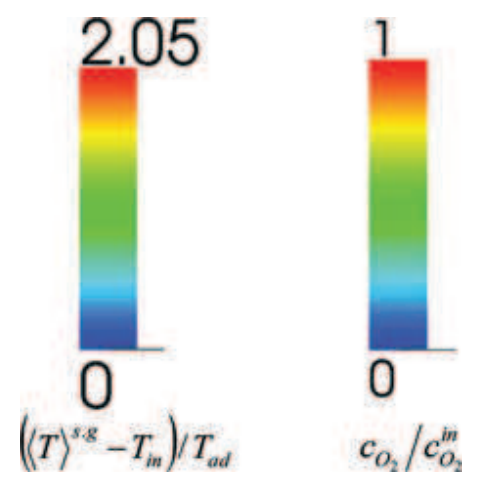

Figure 16 Color codes used in several figures in this section for the display of concentration and temperature.

difference in the macroscale steps from the variation of global effective thermal diffusivity of porous media, which is related to the thermal dispersion and Peclet number etc. More details can be found in Debenest et al. (2005b). The comparisons between the incompressible and compressible flows indicate that the ignorance of the variations of density and dynamic viscosity could result in the obvious discrepancy. Nevertheless, it should be noted that for the low values of $\Delta$, for instance, $\Delta=0.1$ and 0.5 , the agreement between the incompressible and compressible results are acceptable. Since the computations based on the compressible model give rise to the dramatic increase of computation time and complexity, the results based on the incompressible model can be used as the first guess of estimation. 


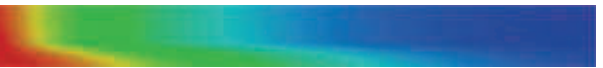

Figure 17 (a) Temperature field when $x_{F}=160 \mathrm{~W}$, for $\Delta=0.5$ and $P e=10$ in the incompressible flow.

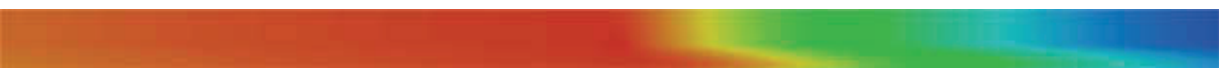

Figure 17 (b) Temperature field when $x_{F}=160 \mathrm{~W}$, for $\Delta=0.5$ and $P e=10$ in the compressible flow.

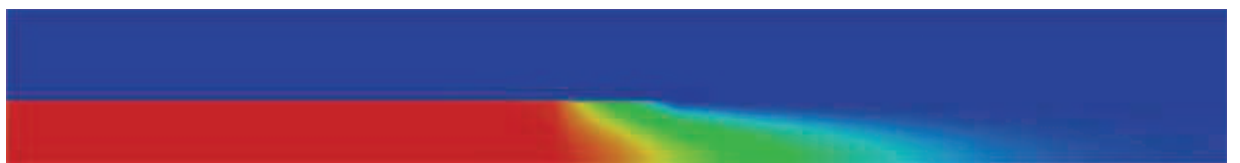

Figure 18 (a) Concentration field when $x_{F}=160 \mathrm{~W}$, for $\Delta=0.5$ and $P e=10$ in the compressible flow.

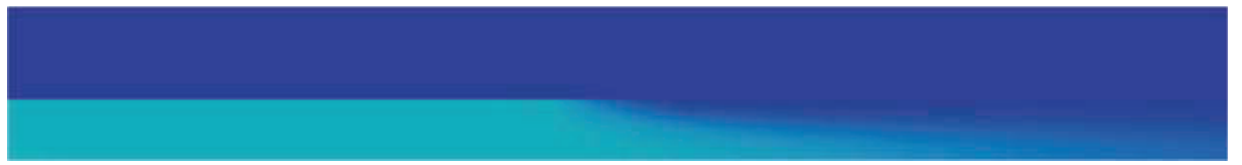

Figure 18 (b) Concentration field when $x_{F}=160 \mathrm{~W}$, for $\Delta=0.5$ and $P e=10$ in the incompressible flow.

Furthermore, the microscopic temperature and oxygen concentration fields are indicated in Figures 17 and 18, for $x_{F}=160 \mathrm{~W}, \Delta=0.5$, and $P e=10$ in the incompressible and compressible flows. Incidentally, Figure 16 shows the color codes used for temperature and oxygen concentration fields for the subsequent figures. As can be seen in these figures, the compressible flow has the larger reaction zone than the incompressible flow does. This phenomenon could be explained by the decay length of oxidizer concentration, which increases with the Peclet number. It should also be noticed that the oxygen concentration of compressible flow is much lower than that of incompressible flow. This is because the temperature increase in the compressible flow would result into the decrease of the mixture density based on Eq. (16).

\section{CONCLUSIONS}

In the present study, numerical simulations were made to investigate the effects of a set of governing parameters on the smoldering process in a horizontal channel. The calculations were carried out for both the compressible and incompressible flows. In the incompressible flow, it was found that a so-called reaction leading structure appears for $\Delta<1$, and $\Delta>1$ results in the reacting trailing structure. Moreover, it should also be noted that for the same $\Delta$, the case of $P e=1$ has a smaller temperature difference between the gas and solid phases than $P e=10$ does, which implies that the local thermal equilibrium assumption could be valid in the case of $P e=1$. By varying the density and dynamic viscosity of the gas phase, the calculations were conducted in the compressible flow to examine the effects of local compressibility and gaseous mixture on the propagation of the smoldering wave. The comparison between the compressible and incompressible flows demonstrates that the variations of pressure, density, and dynamic viscosity of the gas phase in the direction of the smoldering propagation wave are significant. Furthermore, the effects of local compressibility and gaseous mixture on the propagation of smoldering wave are 
negligible only for the small $\Delta$ value, such as $\Delta=0.1$. As the $\Delta$ value increases, the difference of smoldering propagation wave between the compressible and incompressible flows are becoming more noticeable. It can be found that the compressible flow needs a longer distance to reach the equilibrium regime and has the larger reaction zone than the incompressible flow does. Therefore, the considerations of effects of local compressibility and gaseous mixture are necessary to obtain the more realistic and reliable results. As evidenced, the treatment of radiative transfers could buffer some of the conclusions made in this study by decreasing the differences of temperature obtained at the local scale.

\section{FUNDING}

The present research was funded by ANR project (ANR-11-BS009-005-01).

\section{NOMENCLATURE}

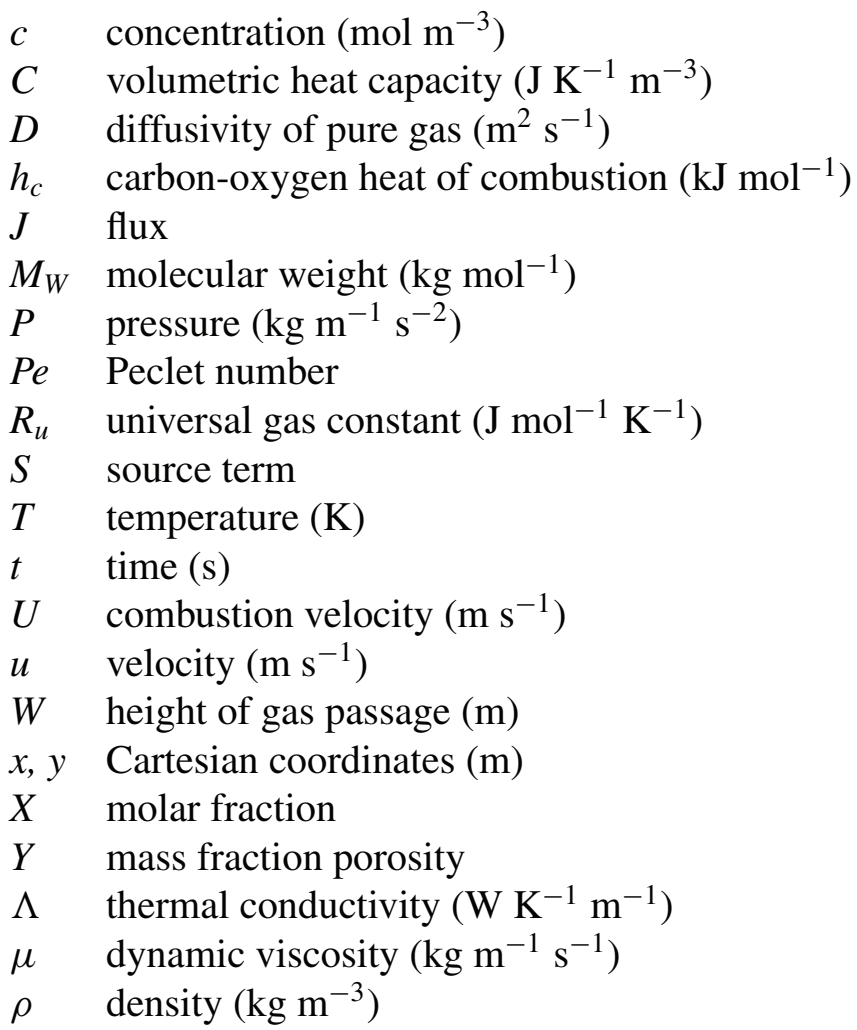

\section{Subscripts}

ad adiabatic

$c$ char

$B$ bulk

$F$ combustion front

$g$ gas

in inlet

$\mathrm{O}_{2}$ oxygen 


$$
\begin{array}{ll}
\mathrm{N}_{2} & \text { nitrogen } \\
\mathrm{CO}_{2} & \text { carbon dioxide } \\
P & \text { plateau } \\
S & \text { solid }
\end{array}
$$

\title{
Superscripts
}

\author{
$g$ gas \\ $s$ solid
}

\section{REFERENCES}

Aldushin, A.P., Merzhanov, A.G., and Seplyarskii, B.S. 1976. Theory of filtration combustion of metals. Fiz Goren Vzryua, 12, 323.

Aldushin, A.P., Rumanov, I.E., and Matkwosky, B.J. 1999. Maximal energy accumulation in a superadiabatic filtration combustion wave. Combust. Flame, 50, 76.

Brenner, G., Pickenäcker, K., Pickenäcker, O., Trimis, D., Wawrzinek, K., and Weber, T. 2000. Numerical and experimental investigation of matrix-stabilized methane/air combustion in porous inert media. Combust. Flame, 23, 201.

Chapman, S., and Cowling, T. 1939. The Mathematical Theory of Non-uniform Gases, Cambridge University Press, New York.

Davit, Y., Debenest, G., Wood, B.D., and Quintard, M. 2010. Modeling non-equilibrium mass transport in biologically reactive porous media. Adv. Water Resour., 33, 1075.

Debenest, G., Mourzenko, V., and Thovert, J.F. 2005a. Smouldering in fixed beds of oil shale grains: A three-dimensional microscale numerical model. Combust. Theor. Model., 9, 113.

Debenest, G., Mourzenko, V., and Thovert, J.F. 2005b. Smouldering in fixed beds of oil shale grains: Governing parameters and global regimes. Combust. Theor. Model., 9, 301.

Debenest, G., Mourzenko, V., and Thovert, J.F. 2008. Three-dimensional microscale numerical simulation of smoldering process in heterogeneous porous media. Combust. Sci. Technol., 180, 2170.

Dosanjh, S.S., Pagni, P.J., and Fernandez-Pello, A.C. 1987. Forced cocurrent smoldering combustion. Combust. Flame, $\mathbf{6 8}, 131$.

Elayeb, M., Debenest, G., Mourzenko, V.V., and Thovert, J.F. 2007. Simulations of smoldering in reactive porous media using a four-species/four-reactions oxidative scheme. Presented at the Eurotherm Meeting, Albi, France, June 4-6.

Hackert, C.L., Ellzey, J.L., and Ezekoye, O.A. 1999. Combustion and heat transfer in model twodimensional porous burners. Combust. Flame, 116, 177.

Lu, M., and Yortsos, Y.C. 2005. The dynamics of forward filtration combustion at the porenetwork level. AIChE J., 51, 1279.

Martins, M.F., Salvador, S., Thovert, J.F., and Debenest, G. 2010a. Co-current combustion of oil shale-Part 1: Characterization of the solid and gaseous products. Fuel, 89, 144.

Martins, M.F., Salvador, S., Thovert, J.F., and Debenest, G. 2010b. Co-current combustion of oil shale-Part 2: Structure of the combustion front. Fuel, 89, 133.

Moallemi, M.K., Zhang, H., and Kumer, S. 1993. Numerical modeling of two-dimensional smoldering processes. Combust. Flame, 95, 170.

Ohlemiller, T.J. 1985. Modeling of smouldering combustion propagation. Prog. Energy Combust. Sci., 11, 277. 
Oliveira, M., and Kaviany, M. 2001. Nonequilibrium in the transport of heat and reactants in combustion in porous media. Prog. Energy Combust. Sci., 27, 523.

Rein, G., Fernandez-Pello, A.C., and Urban, D.L. 2007. Computational model of forward and opposed smoldering combustion in microgravity. Proc. Combust. Inst., 31, 2677.

Sarathi, P.S. 1999. In-Situ Combustion Handbook—Principles and Practices, National Petroleum Technology Office, Tulsa, OK.

Schult, D.A., Matkowsky, B.J., and Volpert, V.A. 1996. Forced forward smolder combustion. Eng. Sci., 2180, 1.

Sennoune, M., Salvador, S., and Quintard, M. 2012. Toward the control of the smoldering front in the reaction-trailing mode in oil shale semicoke porous media. Energy Fuels, 26, 3357.

Tesner, P.A. 1961. The activation energy of gas reactions with solid carbon. Symp. (Int.) Combust., 8, 807.

Torero, J.L., and Fernandez-Pello, A.C. 1996. Forward smolder of polyurethane foam in a forced air flow. Combust. Flame, 106, 89.

Turns, S.R. 1996. An Introduction to Combustion: Concepts and Applications, McGraw-Hill, New York.

Zajdlik, R., Jelemensky, L., Remiarova, B., and Markos, J. 2001. Experimental and modeling investigations of single coal particle combustion. Chem. Eng. Sci., 56, 1355. 